Article

\title{
Multiagent Based Distributed Control with Time-Oriented SoC Balancing Method for DC Microgrid
}

\author{
Tao Wu ${ }^{\circledR}$, Yanghong Xia *®D, Liang Wang and Wei Wei \\ College of Electrical Engineering, Zhejiang University, Hangzhou 310027, China; 21810117@zju.edu.cn (T.W.); \\ 21810215@zju.edu.cn (L.W.); wwei@zju.edu.cn (W.W.) \\ * Correspondence: royxiayh@zju.edu.cn
}

Received: 4 May 2020; Accepted: 29 May 2020; Published: 1 June 2020

check for updates

\begin{abstract}
Based on the droop control, voltage regulation at the secondary control is required to eliminate the deviation of the average voltage across the microgrid. Meanwhile, to prevent any of energy storage (ESs) from over-charging or over-discharging, State-of-Charge (SoC) balancing should be added in the secondary control. This paper proposes a distributed secondary control in the DC microgrid based on the multiagent system (MAS). This controller consists of voltage regulation and time-oriented SoC balancing. In voltage regulation, a PI controller adjusts the droop parameters according to the discrepancy between the average voltage and the reference voltage. In SoC balancing, controller operates in charging mode or discharging mode according to changes of the global average SoC. Being different from the conventional method, the time-oriented SoC balancing method is designed to balance charge/discharge time rather than to balance SoC directly. Thus, SoCs reach a consensus only at the last moment when all ES nodes charge or discharge completely. Furthermore, characteristics, global dynamic model, and steady-state analysis of the proposed control method are studied. Finally, MATLAB/Simulink simulations are performed to verify the effectiveness of the proposed control.
\end{abstract}

Keywords: SoC balance; multiagent system; distributed control; power sharing; secondary control; DC microgrid

\section{Introduction}

In recent years, microgrid has received great attention as an important solution to utilize renewable energy resources such as wind, solar, and tidal energy. Microgrid can effectively integrate distributed generations (DGs), converters, battery energy storage systems (BESSs), and loads. With the advent of more DC loads like electrical vehicles (EVs), DC microgrid has attracted extensive interest and related researches are developing rapidly. Compared to AC microgrid, DC microgrid has considerable advantages. For example, power loss is less without DC/AC converters, moreover, reactive power, frequency, and phase are not problems any more [1,2].

Hierarchical control has been generally accepted since it was put forward [3]. Hierarchical control is composed of primary control, secondary control, and tertiary control. Primary control is the droop control that realizes reference voltage and power sharing. Secondary control eliminates the deviation of voltage and power sharing. Tertiary control is the energy dispatch. According to the configuration of the communication system, secondary control can be divided into 3 types: centralized control, decentralized control, and distributed control. For the centralized control, information like voltages and currents of all nodes including power generation nodes and energy storage (ES) nodes is delivered through network communication to the central controller, then PI controllers calculate droop correction 
terms based on the global information. The most critical disadvantage of the centralized control is that any fault of the network will cause breakdown of the whole secondary control. For the decentralized control, coordination strategy relies on local information without communication links, and its key is to find an appropriate control method to satisfy the requirements of voltage regulation and power sharing. Distributed control is based on multiagent system (MAS), where central controller is not needed anymore and every node only communicates with its adjacent nodes. Controller at every node collects local and neighbors' information to estimate the global average voltage and other information across the whole microgrid by some consensus protocols [4,5].

To prevent any of ESs from over-charging or over-discharging, State-of-Charge (SoC) balancing is necessary. During the discharging process, the ES with higher SoC should supply more power than the others. While during the charging process, the ES with lower SoC should absorb more power than the others. Based on the hierarchical control, SoC balancing methods can be divided into 3 types: centralized, decentralized, and distributed. Through some modified droop control, SoC balancing can be realized in a decentralized way [6-11]. Reference [6] proposed a decentralized control method with SoC balancing. In this method, the droop coefficients are proportional to $S o C^{n}$ during the charging process but are inversely proportional to $S o C^{n}$ during the discharging process. It is obvious that the speed of SoC balancing relies on the parameter $n$. However, there are 3 drawbacks. First, the change ranges of the droop coefficients are too large, which will influence the system stability. Particularly, the system will be unstable when SoC is very small. In addition, the droop coefficients are still changing even after SoC balancing has been achieved. Finally, the method cannot work well when capacities of ESs are different. In Reference [7], a global cost function is proposed based on the effective load and $\mathrm{SoC}$. Then, an optimal droop surface is derived to determine the droop coefficients to balance SoC and optimize the global cost function. In Reference [8], the reference frequency of the droop control is regulated in a linear way according to the changes of local SoC for an AC microgrid, but there will be still frequency deviation during the process of SoC balancing. In Reference [9], the droop coefficients are proportional to $e^{S o C}$ in the charging process but are inversely proportional to $e^{S o C}$ in the discharging process to balance SoC for the DC microgrid. Reference [10] compared various SoC balancing functions in the decentralized control and found that the power function $S o C^{n}$ and the exponential function $e^{S o C}$ were in fact the best choice to promote SoC equalization. Particularly, the power function exhibited the fast speed of SoC equalization and the lowest DC voltage deviation.

For the centralized control, most literatures focused on $\mathrm{SoC}$ balancing by regulating the droop parameters based on the average $\mathrm{SoC}$, like regulating reference currents [11], regulating droop coefficients [12], regulating reference voltages [13], and so on. Generally, control methods used in the centralized control can be also used in the distributed control with some modification. In References [14,15], the droop parameters are adjusted according to the discrepancy between local SoC and the global average SoC, and the latter can be estimated by the dynamic consensus protocol. Furthermore, the droop control can be also adjusted according to the discrepancies among local SoC and neighbors' SoC in References [16,17]. Reference [18] involved sliding mode control in the distributed control of DC microgrid to set the ES's level of participation in the droop control for SoC balancing.

In this paper, a multiagent based distributed control method with voltage regulation and time-oriented SoC balancing for the islanded DC microgrid is proposed. In the novel SoC balancing method, direct control objective is charge/discharge time rather than SoC consensus. In fact, for the SoC balancing, there is no need to make SoCs reach a consensus too early, and it is enough to ensure that ESs charge or discharge completely at the same time. Hence, the charging and discharging time is used as the feedback signals in this paper. Compared to the conventional SoC balancing methods, the proposed time-oriented SoC balancing method can regulate ESs in a more progressive way, which can avoid the ESs with large charging or discharging currents, especially for the extremely unbalanced situations. In this way, the ESs with higher SoC or lower SoC can be protected from overcurrent, and the pressure on filter inductors to avoid saturation is alleviated. Besides, the novel method can work well in 
3 extreme situations where fast discharging and charging is needed, capacities of ESs are different, and variations of line loads are high.

The rest of this paper is organized as follows. First, the preliminaries of MAS and graph theory are introduced in Section 2. Then, Section 3 discusses the global controller including voltage regulation and time-oriented SoC balancing. Besides, Section 4 studies characteristics, global dynamic model, and steady-state analysis of the proposed controller. Furthermore, Section 5 verifies the proposed controller by the islanded DC microgrid simulation. Finally, the paper is concluded in Section 6.

\section{Preliminaries of MAS and Graph Theory}

In MAS, agents cooperate with each other to complete a task. Agents just communicate with their neighbors directly and this sparse communication network can be represented by an undirected graph. In the graph, the nodes are agents, and edges represent the communication links for information exchange. Every node just communicates with its neighbors and makes use of local and neighbors' data to realize global consensus through the average consensus protocol.

The nodes, edges, communication weights, etc. can be described by matrices in the graph. The weighted adjacency matrix is $\mathbf{A}_{\mathbf{G}}=\left[a_{i j}\right] \in \mathbb{R}^{n \times n}$, where $n$ is the number of nodes and $a_{i j}$ denotes communication weight from node $j$ to node $i$. $a_{i j}>0$ when node $j$ is adjacent to node $i$, or $a_{i j}=0$ otherwise. The Laplacian matrix is defined as $\mathbf{L}=\left[l_{i j}\right] \in \mathbb{R}^{n \times n}$, where $l_{i i}=\sum_{j=0}^{n} a_{i j}$ and $l_{i j}=-a_{i j}, i \neq j$. Furthermore, the Laplacian matrix is balanced if the in-degree of each node is equal to its out-degree. For the undirected graph, it has many properties. First, Laplacian matrix of the undirected graph is balanced. Second, if the undirected graph is connected, its $\mathbf{L}$ has a simple zero eigenvalue and all nonzero eigenvalues are positive. Right eigenvector and left eigenvector associated with zero eigenvalue are $\underline{\mathbf{1}}=[1,1, \ldots, 1]^{T}$ and $\underline{\mathbf{1}}^{T}=[1,1, \ldots, 1]$ respectively, in other words, $\mathbf{L} \underline{\mathbf{1}}=\underline{\mathbf{T}}^{T} \mathbf{L}=0$ [19].

\section{Control Layout}

Figure 1 shows the layout of proposed multiagent based distributed control in the DC microgrid, which consists of physical, cyber, and control layers. In the physical layer, there are nodes and remote loads connected to bus. A node consists of ES, power electronic converter including controller like DSP, local loads, and transmission line. In the cyber layer, there is a sparse communication network among all nodes where every node communicates directly to its neighbors. Note that the communication network is nothing to do with the topology of physical microgrid. Importantly, the communication network should be designed such that in case of any link failure, the remaining network is still connected. Ring-like network is a simple but useful example. Generally, links are undirected.

The control layer consists of primary control and secondary control including voltage regulation and time-oriented SoC balancing, which are based on local and neighbors' data. Primary control is mainly composed by the droop control and its control law can be expressed as

$$
v_{i}^{*}=v_{r e f}-r_{i} i_{i}+\delta v_{i}^{1}+\delta v_{i}^{2}
$$

where $\delta v_{i}^{1}$ and $\delta v_{i}^{2}$ mean the first voltage correction term and the second voltage correction term, and they are output of the PI controller $H_{i}$ in voltage regulation and $G_{i}$ in SoC balancing, respectively. Like common droop control, $v_{i}^{*}, v_{r e f}, r_{i}$, and $i_{i}$ are local voltage setpoint, reference voltage, droop coefficient, and current, respectively. 


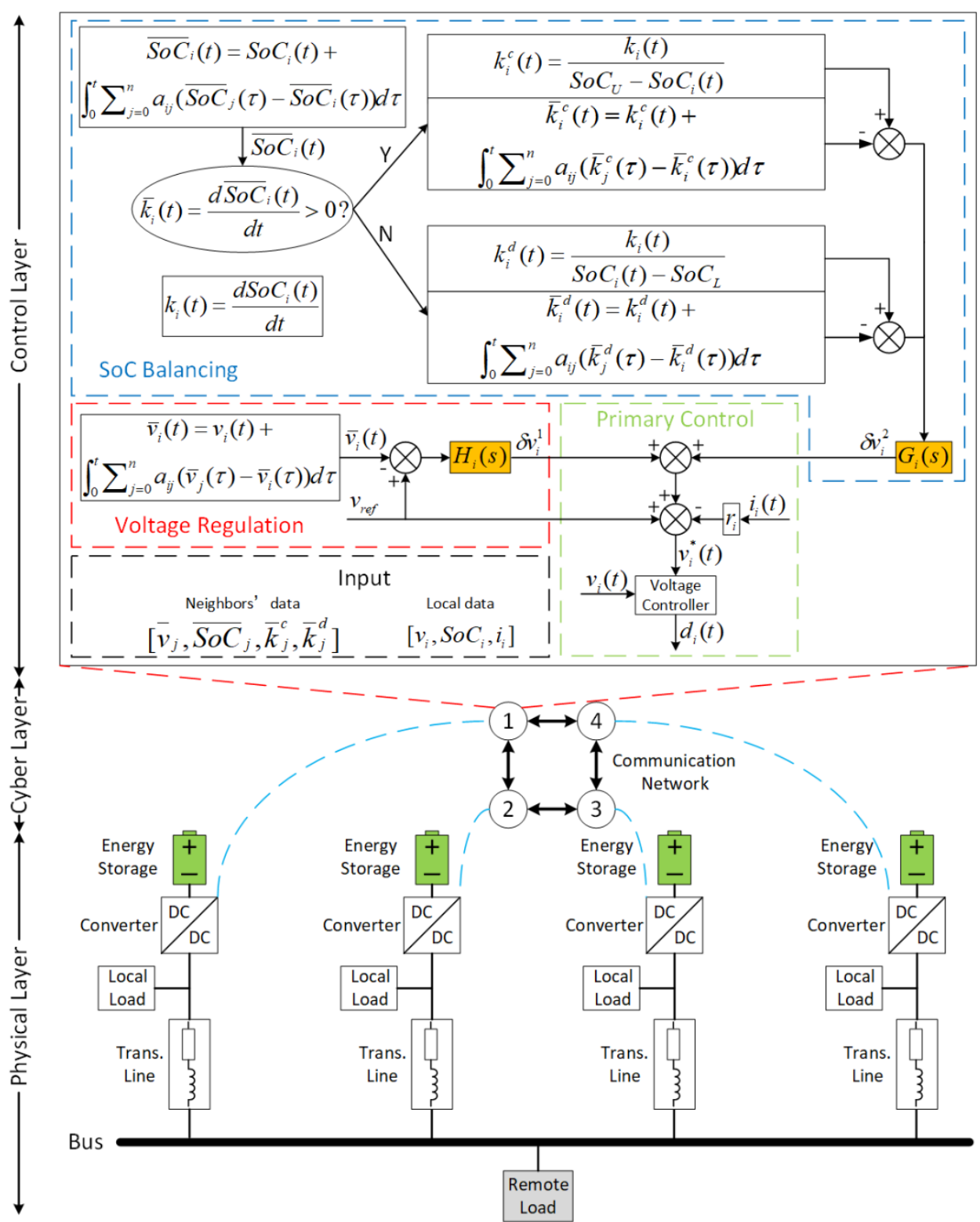

Figure 1. Layout of proposed control for DC microgrid.

\subsection{Voltage Regulation}

As shown in Figure 1, controller at each node estimates the average voltage of the whole microgrid based on local dynamic voltage and neighbors' estimated average voltage, then PI controller $H_{i}$ calculates the first voltage correction term $\delta v_{i}^{1}$ according to the discrepancy between this estimate $\bar{v}_{i}$ and the global reference voltage $v_{\text {ref }}$.

The protocol to estimate the average voltage is designed as

$$
\bar{v}_{i}(t)=v_{i}(t)+\int_{0}^{t} \sum_{j=0}^{n} a_{i j}\left(\bar{v}_{j}(\tau)-\bar{v}_{i}(\tau)\right) d \tau
$$

where $v_{i}(t)$ is the dynamic voltage at node $i . \bar{v}_{j}(t)$ and $\bar{v}_{i}(t)$ denote the estimation of average voltage at node $j$ and node $i$, respectively. By differentiating (2), it can be obtained that

$$
\bar{v}_{i}^{\prime}(t)=v_{i}^{\prime}(t)+\sum_{j=0}^{n} a_{i j}\left(\bar{v}_{j}(t)-\bar{v}_{i}(t)\right)=v_{i}^{\prime}(t)+\sum_{j=0}^{n} a_{i j} \bar{v}_{j}(t)-\sum_{j=0}^{n} a_{i j} \bar{v}_{i}(t)
$$


Rewrite (3) in the matrix form, the following expression can be derived

$$
\overline{\mathbf{v}}^{\prime}=\mathbf{v}^{\prime}-\mathbf{L} \overline{\mathbf{v}}
$$

where $\mathbf{v}=\left[v_{1}, v_{2}, \ldots, v_{n}\right]^{T}$ is the dynamic voltage vector, and $\overline{\mathbf{v}}=\left[\bar{v}_{1}, \bar{v}_{2}, \ldots, \bar{v}_{n}\right]^{T}$ denotes the estimated average voltage vector. The frequency-domain expression of (4) is

$$
s \overline{\mathbf{V}}-\overline{\mathbf{v}}(0)=s \mathbf{V}-\mathbf{v}(0)-\mathbf{L} \overline{\mathbf{V}}
$$

where $\mathbf{V}$ and $\overline{\mathbf{V}}$ are the Laplace transformations of $\mathbf{v}$ and $\overline{\mathbf{v}}$, respectively. According to (2), $\overline{\mathbf{v}}(0)=$ $\mathbf{v}(0)$. Therefore

$$
\overline{\mathbf{V}}=s\left(s \mathbf{I}_{n}+\mathbf{L}\right)^{-1} \mathbf{V}=\mathbf{H}_{o b s} \mathbf{V}
$$

where $\mathbf{I}_{n} \in \mathbb{R}^{n \times n}$ and $\mathbf{H}_{o b s}=s\left(s \mathbf{I}_{n}+\mathbf{L}\right)^{-1}$ are identify matrix and transfer function from $\mathbf{V}$ to $\overline{\mathbf{V}}$, respectively. If $\mathbf{L}$ is balanced, then all elements of $\overline{\mathbf{v}}$ converge to a consensus value, which is the true average voltage, i.e., the average of all elements in $\mathbf{v}$ [5]. In other words

$$
\begin{gathered}
\lim _{t \rightarrow \infty} \mathbf{H}_{o b s}=\mathbf{Q} \\
\overline{\mathbf{v}}^{S S}=\lim _{t \rightarrow \infty} \overline{\mathbf{v}}(t)=\mathbf{Q} \times \lim _{t \rightarrow \infty} \mathbf{v}(t)=\mathbf{Q} \mathbf{v}^{S S}=\left\langle\mathbf{v}^{s S}\right\rangle \underline{\mathbf{1}}
\end{gathered}
$$

where $\mathbf{Q} \in \mathbb{R}^{n \times n}$ is the averaging matrix, whose elements are all equal to $1 / n$. $\mathbf{x}^{\text {ss }}$ expresses the steady-state value of the column vector $\mathbf{x} \in \mathbb{R}^{n \times 1}$. $\langle\mathbf{x}\rangle$ represents the average of all elements in the column vector $\mathbf{x} . \underline{\mathbf{1}} \in \mathbb{R}^{n \times 1}$ is a column vector whose elements are all equal to one.

\subsection{Time-Oriented SoC Balancing Method}

As shown in Figure 1, first, controller at each node estimates the average SoC among all nodes based on local SoC and neighbors' estimated average SoC. According to whether the estimated average SoC is increasing or decreasing, controller operates in charging mode or discharging mode.

The protocol for estimation of average $\mathrm{SoC}$ is designed as

$$
\overline{\operatorname{SoC}}_{i}(t)=\mathrm{SoC}_{i}(t)+\int_{0}^{t} \sum_{j=0}^{n} a_{i j}\left(\overline{\operatorname{SoC}}_{j}(\tau)-\overline{\operatorname{SoC}}_{i}(\tau)\right) d \tau
$$

where $S_{0} C_{i}(t)$ is the real-time SoC at node $i . \overline{S o C}_{j}(t)$ and $\overline{S o C}_{i}(t)$ denote the estimate of global average SoC at node $j$ and node $i$, respectively.

If $\overline{S o C}_{i}(t)$ is increasing, controller will operate in the charging mode, while if $\overline{S o C}_{i}(t)$ is decreasing, controller will operate in the discharging mode.

Generally, according to battery discharge characteristic, SoC of ES should be controlled between the lower limit of $\mathrm{SoC}(20 \%) S_{0} C_{L}$ and the upper limit of SoC (90\%) So $C_{U}$.

\subsubsection{Discharging Mode}

First, the derivative of $S_{0} C_{i}(t)$ can be calculated as

$$
k_{i}(t)=\frac{d S_{0} C_{i}(t)}{d t}
$$

In discharging mode, $k_{i}(t)$ is negative. If $k_{i}(t)$ is constant, then the time for ES at node $i$ to discharge completely can be obtained

$$
t_{i}^{d}=\frac{S_{o} C_{i}(t)-S o C_{L}}{\left|k_{i}(t)\right|}
$$


When $S_{0} C_{i}(t)=S o C_{L}=20 \%$, it means that ES at node $i$ needs to be cut now.

If $t_{i}^{d}>t_{j}^{d}$, it shows that ES at node $i$ is supplying less power than that at node $j$, and it will spend more time for ES at node $i$ to discharge completely. The average completely discharge time among all nodes can be calculated by the average consensus protocol, namely

$$
\bar{t}_{i}^{d}(\mathrm{t})=t_{i}^{d}(t)+\int_{0}^{t} \sum_{j=0}^{n} a_{i j}\left(\bar{t}_{j}^{d}(\tau)-\bar{t}_{i}^{d}(\tau)\right) d \tau
$$

Then $t_{i}^{d}(t)-\bar{t}_{i}^{d}(\mathrm{t})$ can represent the discrepancy of completely discharge time between node $i$ and 'other nodes'. If $t_{i}^{d}(t)-\bar{t}_{i}^{d}(\mathfrak{t})>0$, it shows that ES at node $i$ is supplying less power than others, and the second voltage correction term $\delta v_{i}^{2}$ at node $i$ should increase. Therefore

$$
\delta v_{i}^{2}(t)=g_{i p}\left(t_{i}^{d}(t)-\bar{t}_{i}^{d}(t)\right)+g_{i i} \int\left(t_{i}^{d}(t)-\bar{t}_{i}^{d}(t)\right) d t
$$

where $g_{i p}$ and $g_{i i}$ are the proportional and integral gains, respectively, in the PI controller $G_{i}$.

However, in practice, $k_{i}(t)$ maybe very small and close to $0 . t_{i}^{d}$ will be very large and may be out of limit. In order to use the reciprocal of $t_{i}^{d}$ as the control objective, swap the numerator and denominator in (11) and remove absolute value sign considering $k_{i}(t)<0$. Therefore, (11), (12), and (13) are replaced by

$$
\begin{gathered}
k_{i}^{d}(t)=\frac{k_{i}(t)}{\operatorname{SoC}_{i}(t)-S_{0} C_{L}} \\
\bar{k}_{i}^{d}(t)=k_{i}^{d}(t)+\int_{0}^{t} \sum_{j=0}^{n} a_{i j}\left(\bar{k}_{j}^{d}(\tau)-\bar{k}_{i}^{d}(\tau)\right) d \tau \\
\delta v_{i}^{2}(t)=g_{i p}\left(k_{i}^{d}(t)-\bar{k}_{i}^{d}(t)\right)+g_{i i} \int\left(k_{i}^{d}(t)-\bar{k}_{i}^{d}(t)\right) d t
\end{gathered}
$$

Note that when SoC is close to $\mathrm{SoC}_{L}(20 \%)$, the denominator is close to 0 . So, ES should be cut a little early, for example, when $\mathrm{SoC}=21 \%$.

\subsubsection{Charging Mode}

Protocol in charging mode is very similar to that in discharging mode.

$$
t_{i}^{c}=\frac{S_{o} C_{U}-S o C_{i}(t)}{\left|k_{i}(t)\right|}
$$

This can represent the time for ES at node $i$ to charge completely and $S_{o} C_{i}(t)=S o C_{U}=90 \%$ at that time. Then, the average complete charge time among all nodes can be calculated by the average consensus protocol

$$
\bar{t}_{i}^{c}(t)=t_{i}^{c}(t)+\int_{0}^{t} \sum_{j=0}^{n} a_{i j}\left(\bar{t}_{j}^{c}(\tau)-\bar{t}_{i}^{c}(\tau)\right) d \tau
$$

If $t_{i}^{c}(t)-\bar{t}_{i}^{c}(\mathrm{t})>0$, it shows that ES at node $i$ is absorbing less power than others, and the second voltage correction term at node $i$, i.e., $\delta v_{i}^{2}$, should decrease. Therefore

$$
\delta v_{i}^{2}(t)=g_{i p}\left(\bar{t}_{i}^{c}(t)-t_{i}^{c}(t)\right)+g_{i i} \int\left(\bar{t}_{i}^{c}(t)-t_{i}^{c}(t)\right) d t
$$


In practice, in order to use the reciprocal of $t_{i}^{c}$ as the control objective, swap the numerator and denominator in (17) and remove absolute value sign considering $k_{i}(t)>0$. Therefore, (17), (18), and (19) are replaced by

$$
\begin{gathered}
k_{i}^{c}(t)=\frac{k_{i}(t)}{\operatorname{SoC} C_{U}-\operatorname{SoC}_{i}(t)} \\
\bar{k}_{i}^{c}(t)=k_{i}^{c}(t)+\int_{0}^{t} \sum_{j=0}^{n} a_{i j}\left(\bar{k}_{j}^{c}(\tau)-\bar{k}_{i}^{c}(\tau)\right) d \tau \\
\delta v_{i}^{2}(t)=g_{i p}\left(k_{i}^{c}(t)-\bar{k}_{i}^{c}(t)\right)+g_{i i} \int\left(k_{i}^{c}(t)-\bar{k}_{i}^{c}(t)\right) d t
\end{gathered}
$$

Note that when $\mathrm{SoC}$ is close to $\mathrm{SoC}_{U}(90 \%)$, the denominator is close to 0 . So, ES should be switched to floating charge mode a little early, for example, when SoC $=89 \%$.

The integral of integrator in PI controller $G_{i}$ remains unchanged when controller switches between discharging mode and charging mode, in other words, controller uses the same integrator in both modes. That is because ES with higher SoC always needs to supply more power or to absorb less power, and its $\delta v_{i}^{2}$ is always bigger than that of ES with lower SoC. So, there is no need to clear the integral to zero when controller switches mode.

\section{Characteristics, Global Dynamic Model, and Steady-State Analysis}

\subsection{Characteristics}

As shown in Figure 2, the time-oriented SoC balancing method is designed to balance charge/discharge time rather than to balance SoCs directly, in other words, all ES nodes should charge or discharge completely at the same time. Discrepancies among all SoCs decrease very slowly and SoCs reach a consensus only at the last moment when all ES nodes charge or discharge completely. So, the changing curves of SoCs in this method are milder than those in the traditional method. Therefore, the SoC balancing method can protect ES and its inductors from overcurrent. Apparently, the novel method can work well without knowledge of capacities when capacities of ESs are different.

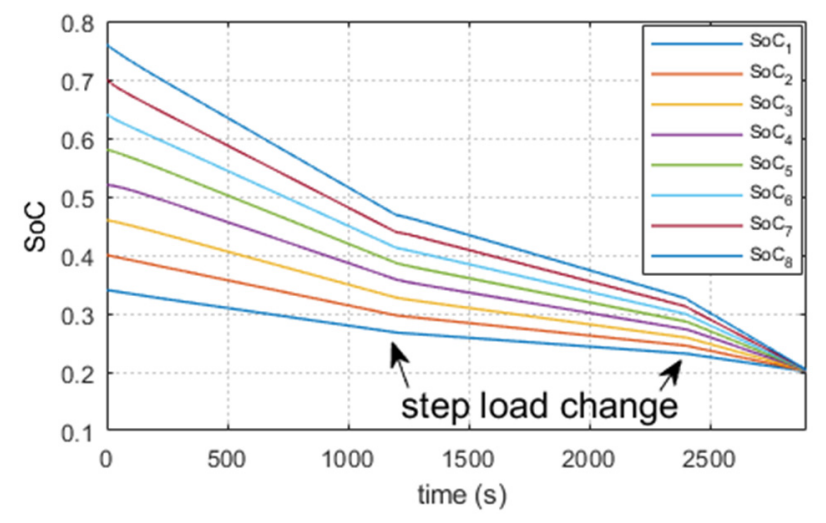

Figure 2. Effects of time-oriented State-of-Charge (SoC) balancing method.

\subsection{Global Dynamic Model}

According to (1), there is

$$
\mathbf{V}^{*}=\mathbf{V}_{\text {ref }}-\mathbf{r I}+\Delta \mathbf{V}^{1}+\Delta \mathbf{V}^{2}
$$

where $\mathbf{V}^{*}=\left[V_{1}^{*}, V_{2}^{*}, \ldots, V_{n}^{*}\right]^{T}, \quad \mathbf{V}_{r e f}=\left[V_{1}^{r e f}, V_{2}^{r e f}, \ldots, V_{n}^{r e f}\right]^{T}, \quad \mathbf{I}=\left[I_{1}, I_{2}, \ldots, I_{n}\right]^{T}, \Delta \mathbf{V}^{1}=$ $\left[\delta V_{1}^{1}, \delta V_{2}^{1}, \ldots, \delta V_{n}^{1}\right]^{T}$, and $\Delta \mathbf{V}^{2}=\left[\delta V_{1}^{2}, \delta V_{2}^{2}, \ldots, \delta V_{n}^{2}\right]^{T}$ are the Laplace transformations of the local voltage set point vector, global reference voltage vector, the dynamic current vector, the first voltage 
correction term vector, and the second voltage correction term vector, respectively. $\mathbf{r}=\operatorname{diag}\left\{r_{i}\right\}$ is the droop coefficient matrix.

$\Delta \mathbf{V}^{1}$ and $\Delta \mathbf{V}^{2}$ can be calculated as

$$
\begin{gathered}
\Delta \mathbf{V}^{1}=\mathbf{H}\left(\mathbf{V}_{r e f}-\overline{\mathbf{V}}\right) \\
\Delta \mathbf{V}^{2}=\mathbf{G}(\mathbf{K}-\overline{\mathbf{K}})
\end{gathered}
$$

where $\mathbf{H}=\operatorname{diag}\left\{H_{i}\right\}$ and $\mathbf{G}=\operatorname{diag}\left\{G_{i}\right\}$ are PI controller matrices of voltage regulation and SoC balancing method, respectively. $\quad K=\left[\frac{K_{1}}{S_{0} C_{1}-S o C_{L}}, \ldots, \frac{K_{n}}{S_{0} C_{n}-S o C_{L}}\right]^{T}$ in discharging mode or $\left[\frac{K_{1}}{\mathrm{SoC}_{U}-S_{0} C_{1}}, \ldots, \frac{K_{n}}{\mathrm{SoC}_{U}-S_{0} C_{n}}\right]^{T}$ in charging mode is the Laplace transformation of SoC balancing variable vector. $\overline{\mathbf{V}}$ and $\overline{\mathbf{K}}$ are the Laplace transformation of estimated average voltage and SoC balancing variable vectors, respectively.

By substituting (24) and (25) in (23)

$$
\mathbf{V}^{*}=\left(\mathbf{I}_{n}+\mathbf{H}\right) \mathbf{V}_{\text {ref }}-\mathbf{r I}-\mathbf{H} \overline{\mathbf{V}}+\mathbf{G}(\mathbf{K}-\overline{\mathbf{K}})
$$

Also, there are

$$
\begin{gathered}
\mathbf{V}=\mathbf{G}_{c} \mathbf{V}^{*} \\
\overline{\mathbf{V}}=\mathbf{H}_{o b s} \mathbf{V} \\
\overline{\mathbf{K}}=\mathbf{H}_{o b s} \mathbf{K} \\
\mathbf{I}=\mathbf{Y V}
\end{gathered}
$$

where $\mathbf{V}=\left[V_{1}, V_{2}, \ldots, V_{2}\right]^{T}$ is the Laplace transformation of dynamic voltage vector, $\mathbf{G}_{c}=\operatorname{diag}\left\{G_{i}^{c}\right\}$ is the closed-loop transfer function matrix of converters, $\mathbf{H}_{o b s}=s\left(s \mathbf{I}_{n}+\mathbf{L}\right)^{-1}$ is the transfer function of dynamic average consensus protocol, and $\mathbf{Y}$ is the global admittance matrix of microgrid.

By substituting (27), (28), (29), and (30) in (26)

$$
\mathbf{V}=\left(\mathbf{G}_{c}^{-1}+\mathbf{r} \mathbf{Y}+\mathbf{H H}_{o b s}\right)^{-1}\left[\left(\mathbf{I}_{n}+\mathbf{H}\right) \mathbf{V}_{\text {ref }}+\mathbf{G}\left(\mathbf{I}_{n}-\mathbf{H}_{o b s}\right) \mathbf{K}\right]
$$

Equation (31) represents the global dynamics of the microgrid under the proposed control methods.

\subsection{Steady-State Analysis}

Here, steady-state analysis is studied to ensure that the proposed controller meets two requirements: the global voltage regulation and the SoC balancing.

First, assume

$$
\mathbf{V}_{r e f}=\frac{v_{r e f}}{s} \underline{\mathbf{1}}
$$

where $v_{r e f}$ is the reference voltage value.

Suppose that dynamic voltage vector $\mathbf{V}$ is stable and the final value theorem is effective. According to (31), there is

$$
\begin{gathered}
\mathbf{v}^{s s}=\lim _{t \rightarrow \infty} \mathbf{v}(t)=\lim _{s \rightarrow 0} \mathbf{V} \mathbf{V}(s) \\
=\lim _{s \rightarrow 0}\left(s \mathbf{G}_{c}^{-1}+s \mathbf{r} \mathbf{Y}+s \mathbf{H H}_{o b s}\right)^{-1}\left[s^{2}\left(\mathbf{I}_{n}+\mathbf{H}\right) \mathbf{V}_{\text {ref }}+s^{2} \mathbf{G}\left(\mathbf{I}_{n}-\mathbf{H}_{o b s}\right) \mathbf{K}\right]
\end{gathered}
$$

It is known that the DC gains of the closed-loop converters are equal to one [5], i.e.,

$$
\mathbf{G}_{c}(0)=\mathbf{I}_{n}=\mathbf{G}_{c}^{-1}(0)
$$


Because $\mathbf{H}$ and $\mathbf{G}$ represent matrices of PI controller coefficients, there are

$$
\begin{aligned}
& \mathbf{H}=\mathbf{H}_{P}+\frac{\mathbf{H}_{I}}{s} \\
& \mathbf{G}=\mathbf{G}_{P}+\frac{\mathbf{G}_{I}}{s}
\end{aligned}
$$

where $\mathbf{H}_{P}$ and $\mathbf{H}_{I}$ denote diagonal matrices carrying proportional and integral gains of the voltage controllers. Similarly, $\mathbf{G}_{P}$ and $\mathbf{G}_{I}$ denote diagonal matrices carrying proportional and integral gains of the time-oriented SoC balancing controllers.

By substituting (7), (32), (34), (35), and (36) in (33)

$$
\begin{gathered}
\mathbf{v}^{s S}=\left(\mathbf{H}_{I} \mathbf{Q}\right)^{-1}\left[v_{r e f} \mathbf{H}_{I} \underline{\mathbf{1}}+\mathbf{G}_{I}\left(\mathbf{I}_{n}-\mathbf{Q}\right) \mathbf{K}^{s s}\right] \\
\mathbf{H}_{I} \mathbf{Q} \mathbf{v}^{s s}=v_{r e f} \mathbf{H}_{I} \underline{\mathbf{1}}+\mathbf{G}_{I}\left(\mathbf{I}_{n}-\mathbf{Q}\right) \mathbf{K}^{s s}
\end{gathered}
$$

All the integrator gains for the SoC balancing controllers are positive, so, $\mathrm{G}_{I}^{-1}$ exists. Pre-multiply at both sides of (38) by $\mathbf{Q G}_{I}^{-1}$

$$
\mathbf{Q U Q \mathbf { Q }} \mathbf{v}^{s s}=v_{r e f} \mathbf{Q U} \underline{\mathbf{1}}+\mathbf{Q}\left(\mathbf{I}_{n}-\mathbf{Q}\right) \mathbf{K}^{s S}
$$

where $\mathbf{U}=\mathbf{G}_{I}^{-1} \mathbf{H}_{I}=\operatorname{diag}\left\{u_{i}\right\}=\operatorname{diag}\left\{\frac{h_{i}}{g_{i}}\right\}$. Because $\mathbf{Q}$ is the averaging matrix, whose elements are all equal to $1 / n$, there is

$$
\mathbf{Q Q}=\mathbf{Q}
$$

By substituting (40) in (39)

$$
\mathbf{Q}\left(\mathbf{U}\left(\mathbf{Q v}^{s s}\right)\right)=v_{r e f} \mathbf{Q}(\mathbf{U} \underline{\mathbf{1}})
$$

Based on the definition of $\mathbf{Q}, \mathbf{Q x}=\langle\mathbf{x}\rangle \underline{\mathbf{1}}$, Equation (41) is equivalent to

$$
\left\langle\mathbf{v}^{s s}\right\rangle\langle\mathbf{U} \underline{\mathbf{1}}\rangle \underline{\mathbf{1}}=v_{r e f}\langle\underline{\mathbf{U}}\rangle \underline{\mathbf{1}}
$$

Therefore

$$
\left\langle\mathbf{v}^{s s}\right\rangle=v_{\text {ref }}
$$

It shows that the average voltage across the microgrid can be regulated at the reference voltage value as expected.

Therefore, it can be derived from Equation (38) that

$$
\mathbf{G}_{I}\left(\mathbf{I}_{n}-\mathbf{Q}\right) \mathbf{K}^{S S}=0
$$

Pre-multiply at both sides of (44) by $\mathbf{L G}_{I}^{-1}$

$$
(\mathbf{L}-\mathbf{L Q}) \mathbf{K}^{S \mathcal{S}}=0
$$

Because $\mathbf{L} \underline{\mathbf{1}}=0, \mathbf{L} \mathbf{Q}=0$. Therefore, (45) can be written as

$$
\mathbf{L K}^{S \mathcal{S}}=0
$$

Therefore, $\mathbf{K}^{s s}$ is the right eigenvector of $\mathbf{L}$ associated with zero eigenvalue, and

$$
\mathbf{K}^{s S}=m \underline{\mathbf{1}}
$$

where $m$ is a scalar factor. It implies that the time for all ESs to charge or discharge completely is identical. 


\section{Simulation Results}

An islanded 400V DC microgrid comprised of 8 ES nodes is simulated in MATLAB/Simulink to verify the effectiveness of proposed controller. Eight ES nodes and a remote load are connected in parallel with bus physically, and a communication network of 8 nodes is shown in Figure 3 . Battery at each node is connected to bus through bidirectional Buck-Boost converter. Transmission line impedances at both sides are identical. Public and private parameters of 8 ES nodes are shown in Tables 1 and 2, respectively.

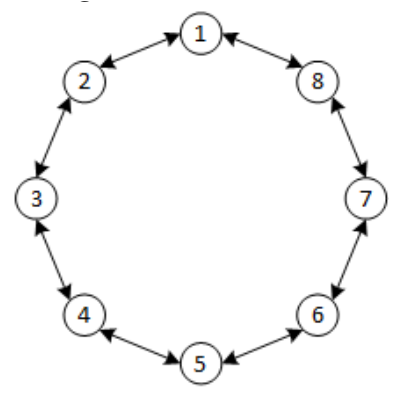

Figure 3. Communication network.

Table 1. Public parameters of 8 nodes.

\begin{tabular}{cc}
\hline Parameters & Value \\
\hline Battery nominal voltage & $144 \mathrm{~V}$ \\
\hline Converter inductance & $0.01 \mathrm{H}$ \\
\hline Converter capacitance & $1000 \mathrm{uF}$ \\
\hline Reference voltage & $400 \mathrm{~V}$ \\
\hline Droop coefficient & 1 \\
\hline PWM frequency & $10 \mathrm{kHz}$ \\
\hline Secondary control period & $0.001 \mathrm{~s}$ \\
\hline Voltage regulation PI proportional & 1 \\
\hline Voltage regulation PI integral & 50 \\
\hline SoC balancing PI proportional & 3000 \\
\hline SoC balancing PI integral & 30,000 \\
\hline Lower limit of SoC & $20 \%$ \\
\hline Upper limit of SoC & $90 \%$ \\
\hline
\end{tabular}

Table 2. Private parameters of 8 nodes.

\begin{tabular}{ccccccccc}
\hline Parameters & Node 1 & Node 2 & Node 3 & Node 4 & Node 5 & Node 6 & Node 7 & Node 8 \\
\hline Rated capacity (Ah) & 80 & 40 & 40 & 80 & 80 & 40 & 40 & 80 \\
\hline Initial SoC & $34 \%$ & $40 \%$ & $46 \%$ & $52 \%$ & $58 \%$ & $64 \%$ & $70 \%$ & $76 \%$ \\
\hline $\begin{array}{c}\text { Transmission line } \\
\text { impedance at each side } \\
\text { (Ohms) }\end{array}$ & 0.4 & 0.3 & 0.25 & 0.2 & 0.15 & 0.1 & 0.05 & 0.01 \\
\hline
\end{tabular}

Communication weight $a_{i j}$ is chosen by an improved Metropolis method [20]

$$
a_{i j}=\left\{\begin{array}{c}
\frac{1}{\max \left(n_{i}, n_{j}\right)+1}, j \text { is a neighbor of } i \\
0, \text { otherwise }
\end{array}\right.
$$


where $n_{i}$ denotes the number of neighbors at node $i$.

A total of 5 cases are simulated and results are analyzed. The first case is charging case, the second case is discharging case, the third case is switching case, the fourth case is fast discharging and charging case, and the last case is the comparative case using the conventional $\mathrm{SoC}$ balancing method proposed in Reference [6].

- Case 1: Charging simulation

Charging simulation is performed for $3000 \mathrm{~s}$. The average voltage across the microgrid is shown in Figure 4a. Dynamic voltages of 8 ES nodes are shown in Figure 4b. SoCs of 8 ES nodes are shown in Figure 4c. Output currents of 8 nodes are shown in Figure $4 \mathrm{~d}$. At first, droop controller is implemented, remote load is $8 \Omega$, and an extra constant current source supplies 150A current. At $t=1 \mathrm{~s}$, proposed controller is performed. At $t=1200 \mathrm{~s}$, remote load changes in step from $8 \Omega$ to $4 \Omega$. At $t=2400 \mathrm{~s}$, remote load changes in step from $4 \Omega$ to $8 \Omega$.

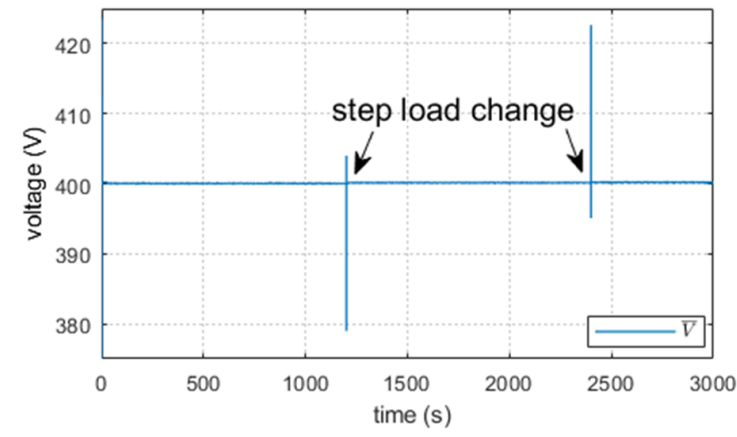

(a)

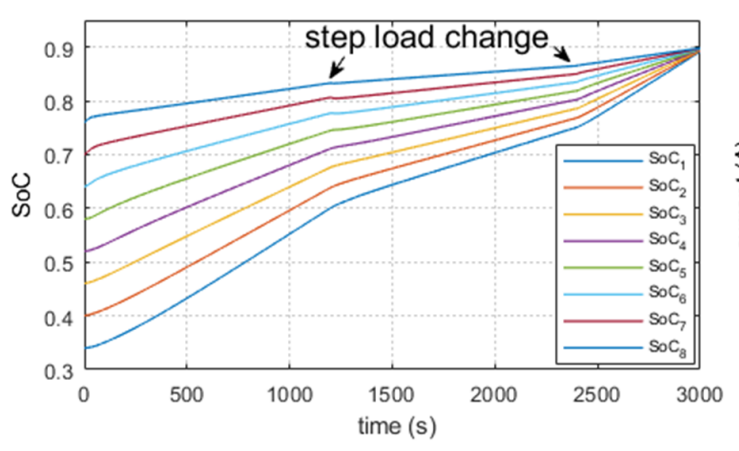

(c)

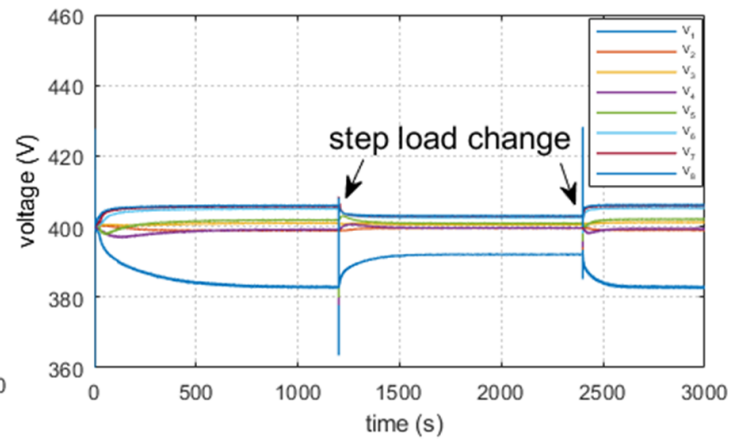

(b)

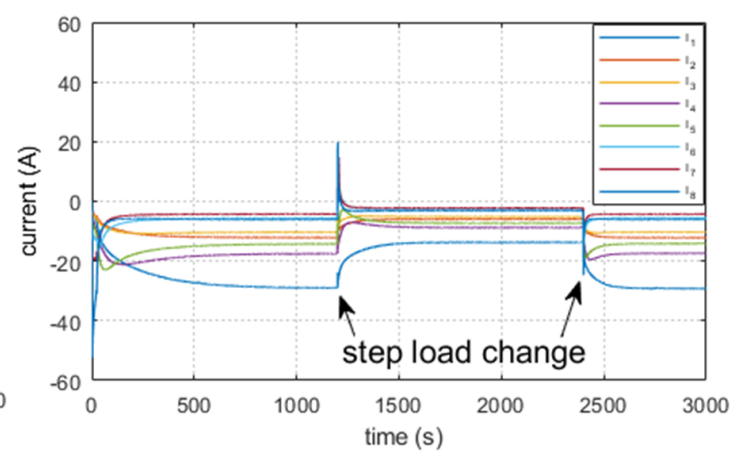

(d)

Figure 4. Discharging simulation results: (a) average voltage; (b) dynamic voltages; (c) SoCs; (d) output currents.

It can be seen that SoCs of $8 \mathrm{ES}$ nodes have been different until all SoCs are equal to $90 \%$.

- $\quad$ Case 2: Discharging simulation

Discharging simulation is performed for $2900 \mathrm{~s}$. The average voltage across the microgrid is shown in Figure 5a. Dynamic voltages of 8 ES nodes are shown in Figure 5b. SoCs of 8 ES nodes are shown in Figure 5c. Output currents of 8 nodes are shown in Figure 5d. At first, droop controller is implemented and remote load is $4 \Omega$. At $t=1 \mathrm{~s}$, proposed controller is performed. At $t=1200 \mathrm{~s}$, remote load changes in step from $4 \Omega$ to $8 \Omega$. At $t=2400 \mathrm{~s}$, remote load changes in step from $8 \Omega$ to $4 \Omega$. 


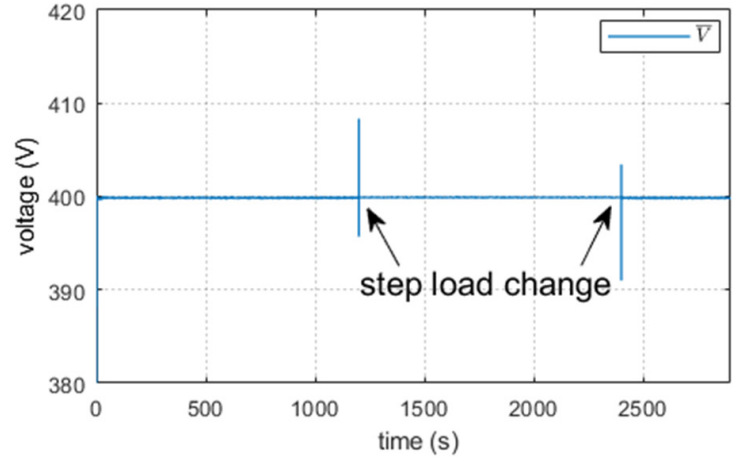

(a)

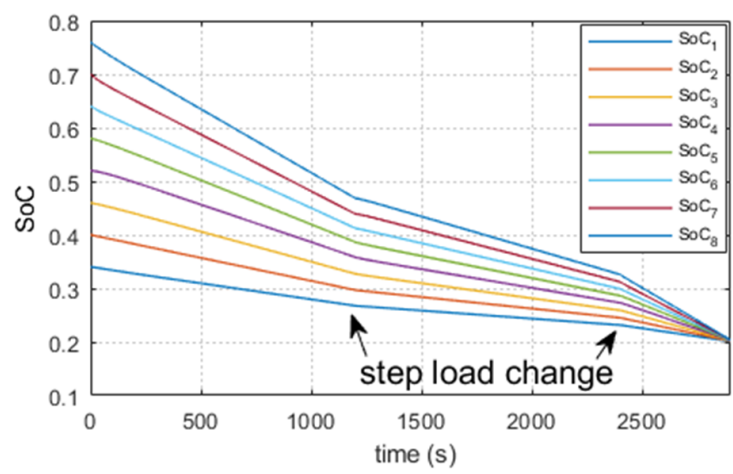

(c)

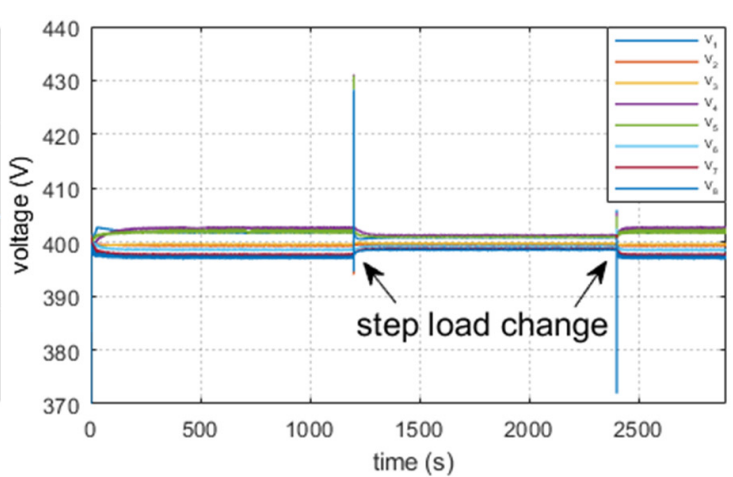

(b)

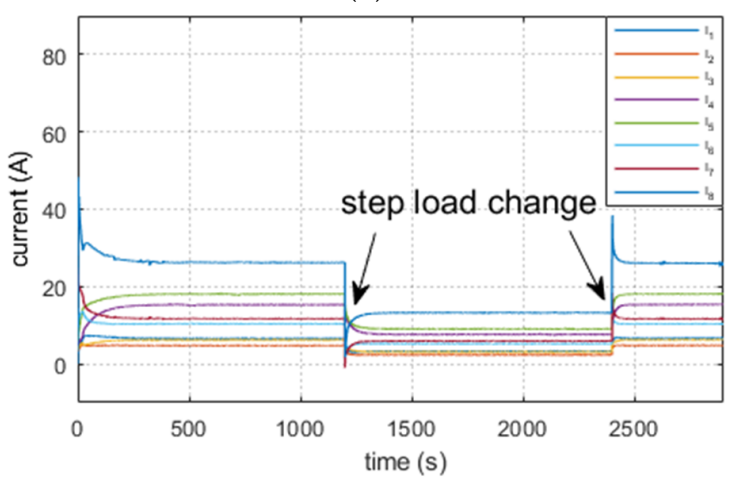

(d)

Figure 5. Charging simulation results: (a) average voltage; (b) dynamic voltages; (c) SoCs; (d) output currents.

It can be seen that SoCs of 8 ES nodes have been different until all SoCs are equal to $20 \%$.

- $\quad$ Case 3: Switching the status of operation from discharging mode to charging mode

Switching simulation is performed for $3800 \mathrm{~s}$. The average voltage across the microgrid is shown in Figure 6a. Dynamic voltages of 8 ES nodes are shown in Figure 6b. SoCs of 8 ES nodes are shown in Figure 6c. Output currents of 8 nodes are shown in Figure 6d. At first, droop controller is implemented and remote load is $8 \Omega$. Eight ES nodes are in discharging mode. At $t=1 \mathrm{~s}$, proposed controller is performed. At $t=500 \mathrm{~s}$, an extra constant current source is connected to bus and supplies $150 \mathrm{~A}$ current. Eight ES nodes are switched to charging mode according to the average SoC increasing at this time. At $t=1700 \mathrm{~s}$, remote load changes in step from $8 \Omega$ to $4 \Omega$. At $t=3000 \mathrm{~s}$, remote load changes in step from $4 \Omega$ to $8 \Omega$.

It can be seen that, according to whether the average SoC is increasing or decreasing, controllers switch between discharging mode and charging mode smoothly.

- $\quad$ Case 4: Fast discharging and charging simulation

Fast discharging and charging simulation are performed for 425s. Compared to previous 3 cases, capacities of all ESs and event time are different in the case. The capacities of 8 ESs are shown in Table 3. The average voltage across the microgrid is shown in Figure 7a. Dynamic voltages of 8 ES nodes are shown in Figure 7b. SoCs of 8 ES nodes are shown in Figure 7c. Output currents of 8 nodes are shown in Figure $7 d$. At first, droop controller is implemented and remote load is $8 \Omega$. A total of 8 ES nodes are in discharging mode. At $t=1 \mathrm{~s}$, proposed controller is performed. At $t=50 \mathrm{~s}$, an extra constant current source is connected to bus and supplies 150 A current. Eight ES nodes are switched to charging mode according to the average SoC increasing at this time. At $t=170 \mathrm{~s}$, remote load changes in step from $8 \Omega$ to $4 \Omega$. At $t=320 \mathrm{~s}$, remote load changes in step from $4 \Omega$ to $8 \Omega$. 


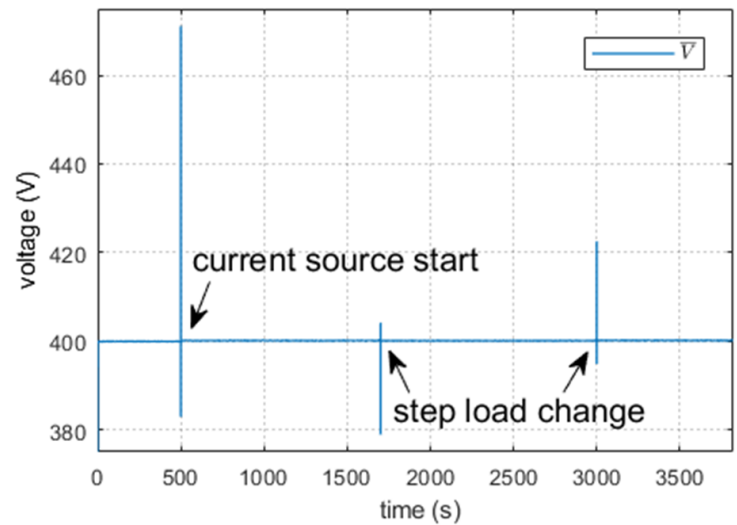

(a)

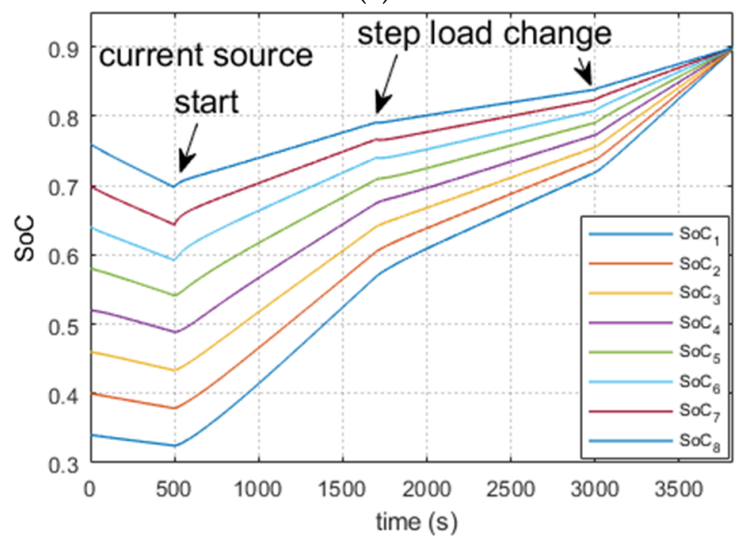

(c)

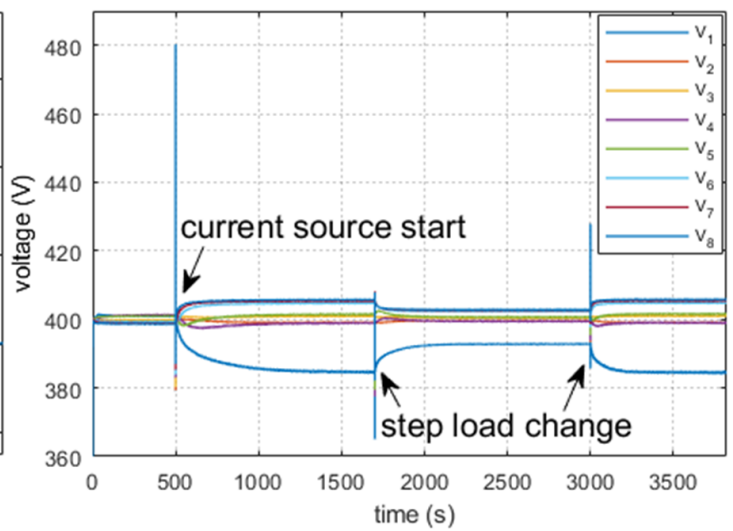

(b)

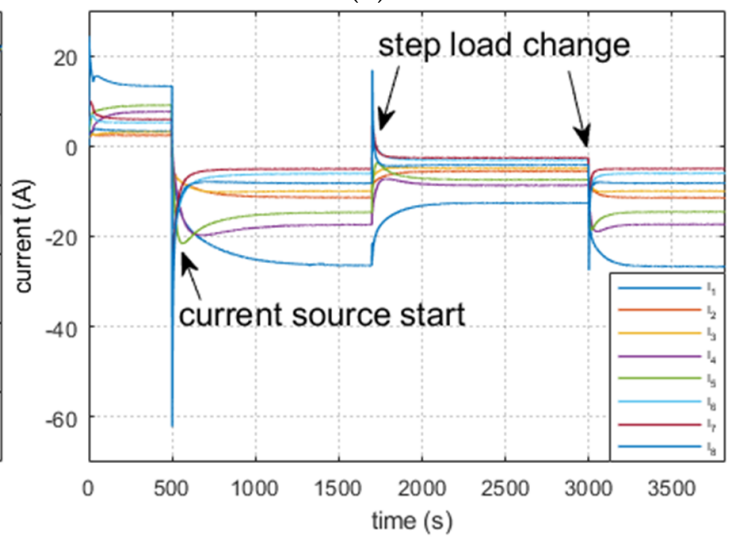

(d)

Figure 6. Switching simulation results: (a) average voltage; (b) dynamic voltages; (c) SoCs; (d) output currents.

Table 3. Capacities of $8 \mathrm{ESs}$ in fast discharging and charging simulation.

\begin{tabular}{ccccccccc}
\hline Parameters & Node 1 & Node 2 & Node 3 & Node 4 & Node 5 & Node 6 & Node 7 & Node 8 \\
\hline Rated capacity (Ah) & 8 & 4 & 4 & 8 & 8 & 4 & 4 & 8 \\
\hline
\end{tabular}

It can be seen that the time-oriented SoC balancing method can work well in fast discharging and charging situation.

- $\quad$ Case 5: Comparative simulation using the conventional SoC balancing method

The all basic parameters for simulation in the case are the same as case 4 . The protocol for SoC balancing is chosen as

$$
\text { droop coefficient } r_{i}=\left\{\begin{array}{c}
\frac{20}{S o C_{i}^{5}}, \text { discharging } \\
20 \times S o C_{i}^{5} \text {, charging }
\end{array}\right.
$$

The voltage at the remote load is shown in Figure 8a. Dynamic voltages of 8 ES nodes are shown in Figure 8 b. SoCs of 8 ES nodes are shown in Figure 8c. Output currents of 8 nodes are shown in Figure 8d. 


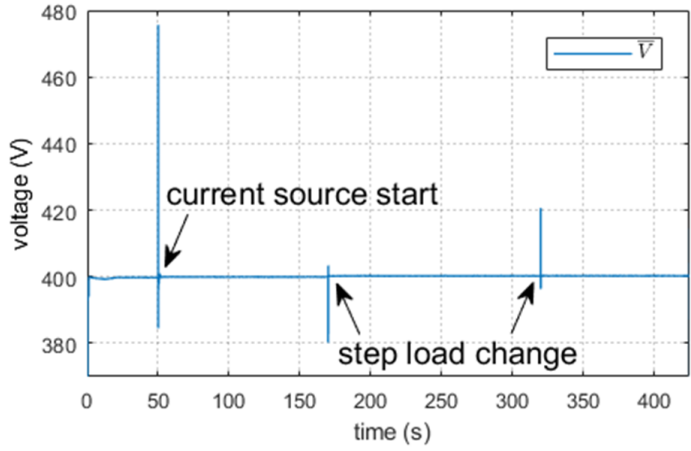

(a)

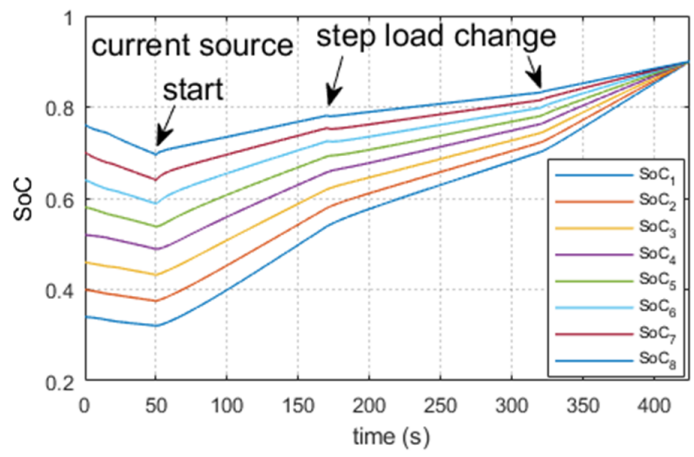

(c)

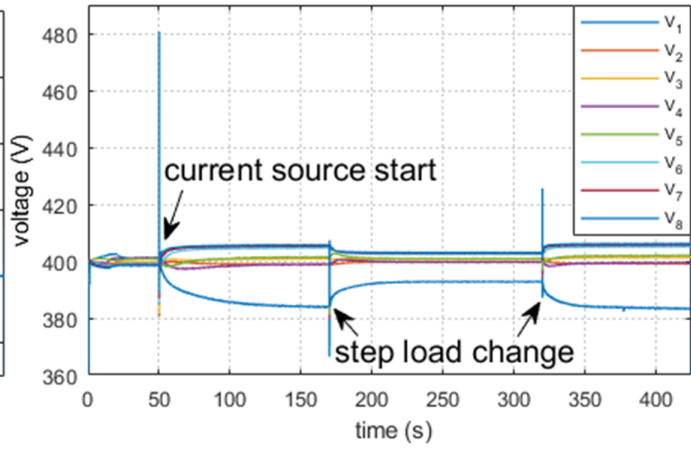

(b)

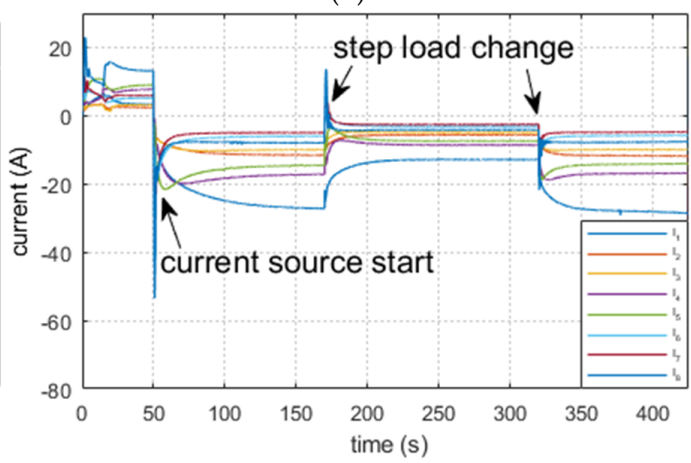

(d)

Figure 7. Fast discharging and charging simulation results: (a) average voltage; (b) dynamic voltages; (c) SoCs; (d) output currents.

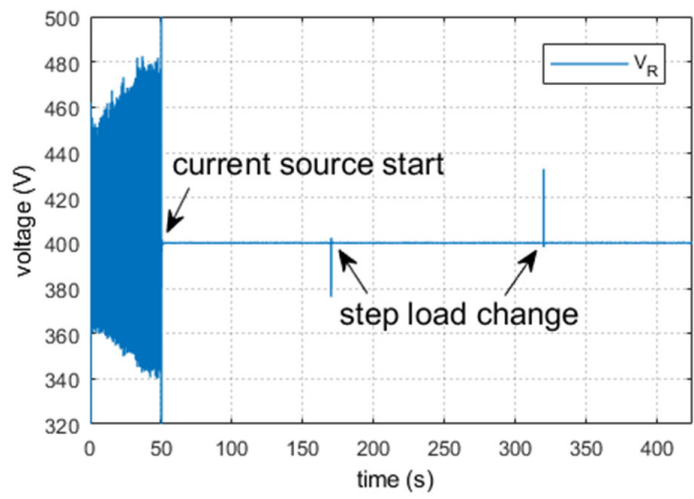

(a)

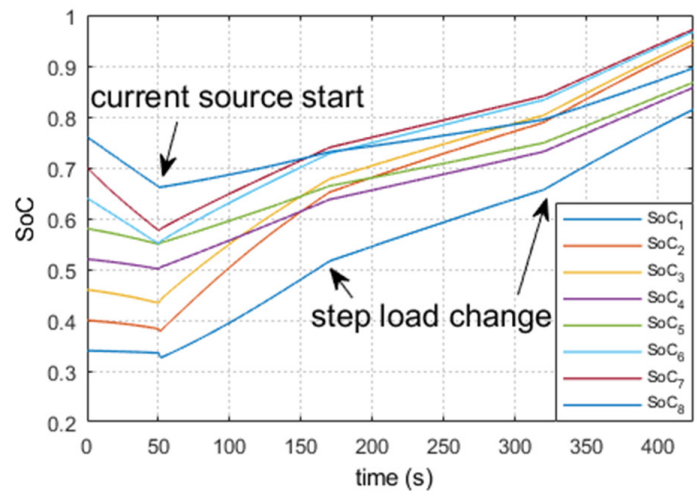

(c)

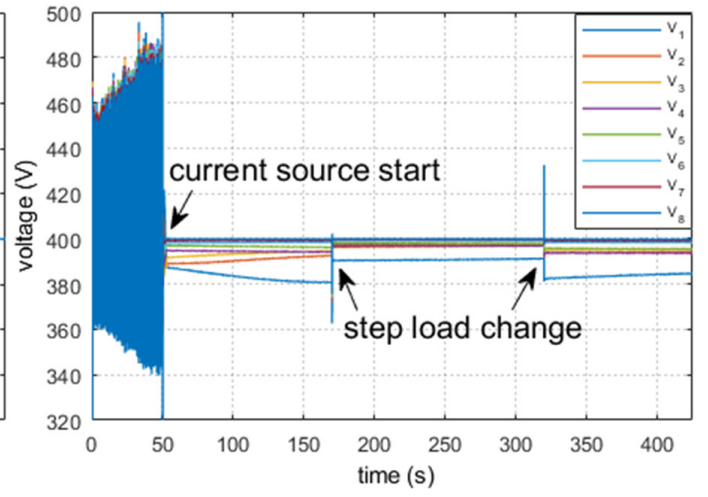

(b)

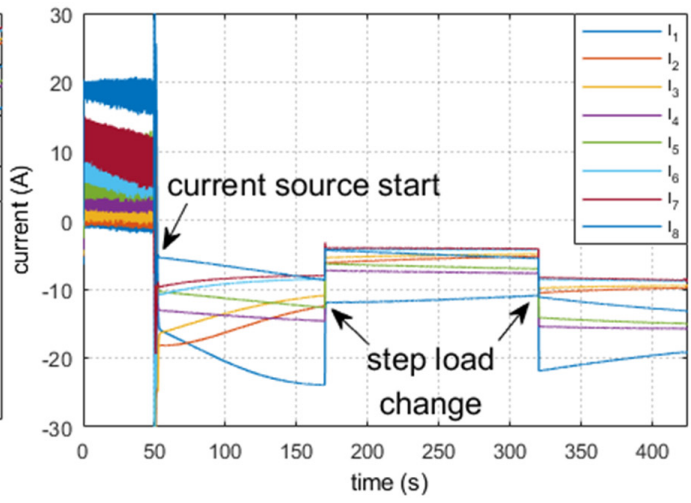

(d)

Figure 8. Comparative simulation results: (a) voltage at the remote load; (b) dynamic voltages; (c) SoCs; (d) output currents. 
It can be seen that the SoC balancing method proposed in Reference [6] has 3 drawbacks. First, voltage fluctuates widely in the discharging process because the droop coefficients are too big, which is caused by $\frac{1}{\mathrm{SoC}^{n}}$. Second, the speed of SoC balancing is too slow compared to time-oriented SoC balancing method, though $n=5$ in $r_{i}=20 \times S o C_{i}^{n}$. This is because the conventional SoC balancing method cannot work well when the variations of line loads are very high. Third, the conventional SoC balancing method cannot work well when capacities of ESs are different.

Last but not least, if the speed of $\mathrm{SoC}$ balancing in the conventional method is faster than that in the time-oriented SoC balancing method, the peak currents in the conventional method will be higher.

Five cases can verify the effectiveness of the proposed controller very well. Apparently, voltage deviation between the reference voltage and the average voltage has been evidently eliminated after the proposed controller is performed. There are always differences among dynamic voltage of $8 \mathrm{ES}$ nodes, but the average voltage across the microgrid is stabilized at reference voltage. Furthermore, all SoCs have been different until they are all equal to $90 \%$ in charging mode or $20 \%$ in discharging mode. Besides, proposed controllers switch between charging mode and discharging mode smoothly.

It can be seen that the time-oriented SoC balancing method can work well in 3 extreme situations where fast discharging and charging is needed, capacities of ESs are different, and variations of line loads are high.

It is worth mentioning that output currents are almost constant without step load change during SoC balancing. However, output currents will change when SoC balance is achieved in the conventional SoC balancing methods.

\section{Conclusions}

A multiagent based distributed controller in DC microgrid is proposed. The controller consists of voltage regulation and time-oriented SoC balancing. Voltage regulator estimates average voltage, and the discrepancy between this estimation and reference voltage is then used to calculate the first voltage correction term by PI controller. In the SoC balancing, the average SoC needs to be estimated first. If the average $\mathrm{SoC}$ is increasing, the controller will be in charging mode, or the controller will be in discharging mode otherwise. The time-oriented SoC balancing method balances charge/discharge time rather than SoCs directly; in other words, all ES nodes should charge or discharge completely at the same time. Global dynamic model and steady-state analysis are studied, and MATLAB/Simulink simulations verify the proposed controller. The advantages of the novel method are summarized as follows. First, the method can work well without knowledge of capacities when capacities of ESs are different. Second, the method does a good job in fast discharging and charging process. Third, the method is not sensitive to line loads. Fourth, the method can protect the ES and filter inductor from overcurrent.

Author Contributions: Conceptualization, T.W.; methodology, T.W.; software, T.W.; validation, T.W.; formal analysis, T.W.; investigation, T.W.; resources, Y.X.; data curation, L.W.; writing-original draft preparation, T.W.; writing-review and editing, Y.X.; visualization, Y.X.; supervision, Y.X.; project administration, W.W.; funding acquisition, W.W. All authors have read and agreed to the published version of the manuscript.

Funding: This research was funded by the Project founded by the China Postdoctoral Science Foundation (2019M660139) and the National Key R\&D Program of China (2017YFB0903300).

Conflicts of Interest: The authors declare no conflict of interest. 


\section{References}

1. Dragičević, T.; Lu, X.; Vasquez, J.C.; Guerrero, J.M. DC Microgrids-Part I: A Review of Control Strategies and Stabilization Techniques. IEEE Trans. Power Electron. 2016, 31, 4876-4891. [CrossRef]

2. Dragičević, T.; Lu, X.; Vasquez, J.C.; Guerrero, J.M. DC Microgrids-Part II: A Review of Power Architectures, Applications, and Standardization Issues. IEEE Trans. Power Electron. 2016, 31, 3528-3549. [CrossRef]

3. Guerrero, J.M.; Vasquez, J.C.; Matas, J.; Vicuna, L.G.d.; Castilla, M. Hierarchical Control of Droop-Controlled AC and DC Microgrids-A General Approach Toward Standardization. IEEE Trans. Ind. Electron. 2011, 58, 158-172. [CrossRef]

4. Nasirian, V.; Davoudi, A.; Lewis, F.L.; Guerrero, J.M. Distributed Adaptive Droop Control for DC Distribution Systems. IEEE Trans. Energy Convers. 2014, 29, 944-956. [CrossRef]

5. Nasirian, V.; Moayedi, S.; Davoudi, A.; Lewis, F.L. Distributed Cooperative Control of DC Microgrids. IEEE Trans. Power Electron. 2015, 30, 2288-2303. [CrossRef]

6. Lu, X.; Sun, K.; Guerrero, J.M.; Vasquez, J.C.; Huang, L. Double-Quadrant State-of-Charge-Based Droop Control Method for Distributed Energy Storage Systems in Autonomous DC Microgrids. IEEE Trans. Smart Grid 2015, 6, 147-157. [CrossRef]

7. Jones, A.J.; Weaver, W.W. Optimal Droop Surface Control of dc Microgrids Based on Battery State of Charge. In Proceedings of the 2016 IEEE Energy Conversion Congress and Exposition (ECCE), Milwaukee, WI, USA, 18-22 September 2016; pp. 1-8.

8. Wu, Q.; Guan, R.; Sun, X.; Wang, Y.; Li, X. SoC Balancing Strategy for Multiple Energy Storage Units With Different Capacities in Islanded Microgrids Based on Droop Control. IEEE J. Emerg. Sel. Top. Power Electron. 2018, 6, 1932-1941. [CrossRef]

9. Dragičević, T.; Guerrero, J.M.; Vasquez, J.C.; Škrlec, D. Supervisory Control of an Adaptive-Droop Regulated DC Microgrid With Battery Management Capability. IEEE Trans. Power Electron. 2014, 29, 695-706. [CrossRef]

10. Marcelino, F.L.F.; Sathler, H.H.; Silva, W.W.A.G.; de Oliveira, T.R.; Donoso-Garcia, P.F. A Comparative Study of Droop Compensation Functions for State-of-Charge Based Adaptive Droop Control for Distributed Energy Storage Systems. In Proceedings of the 2017 IEEE 8th International Symposium on Power Electronics for Distributed Generation Systems (PEDG), Florianopolis, Brazil, 17-20 April 2017; pp. 1-8.

11. Gkavanoudis, S.I.; Oureilidis, K.O.; Kryonidis, G.C.; Demoulias, C.S. A Control Method for Balancing the SoC of Distributed Batteries in Islanded Converter-Interfaced Microgrids. Adv. Power Electron. 2016, 2016, 1-11. [CrossRef]

12. Palizban, O.; Kauhaniemi, K. Distributed cooperative control of battery energy storage system in AC microgrid applications. J. Energy Storage 2015, 3, 43-51. [CrossRef]

13. Chendan, L.; Dragicevic, T.; Diaz, N.L.; Vasquez, J.C.; Guerrero, J.M. Voltage Scheduling Droop Control for State-of-Charge Balance of Distributed Energy Storage in DC Microgrids. In Proceedings of the 2014 IEEE International Energy Conference (ENERGYCON), Cavtat, Croatia, 13-16 May 2014; pp. 1310-1314.

14. Li, C.; Dragicevic, T.; Plaza, M.G.; Andrade, F.; Vasquez, J.C.; Guerrero, J.M. Multiagent Based Distributed Control for State-of-Charge Balance of Distributed Energy Storage in DC Microgrids. In Proceedings of the IECON 2014 - 40th Annual Conference of the IEEE Industrial Electronics Society, Dallas, TX, USA, 29 October-1 November 2014; pp. 2180-2184.

15. Li, C.; Coelho, E.A.A.; Dragicevic, T.; Guerrero, J.M.; Vasquez, J.C. Multiagent-Based Distributed State of Charge Balancing Control for Distributed Energy Storage Units in AC Microgrids. IEEE Trans. Ind. Appl. 2017, 53, 2369-2381. [CrossRef]

16. Shi, G.; Sun, Y.; Han, H.; Su, M.; Hou, X.; Luo, Z. A Unified SoC Balancing Method with Low-Bandwidth Distributed Communication in Island Microgrid. In Proceedings of the 2017 IEEE 3rd International Future Energy Electronics Conference and ECCE Asia (IFEEC 2017_ECCE Asia), Kaohsiung, Taiwan, 3-7 June 2017; pp. 573-578.

17. Chen, X.; Shi, M.; Zhou, J.; Chen, Y.; Zuo, W.; Wen, J.; He, H. Distributed Cooperative Control of Multiple Hybrid Energy Storage Systems in a DC Microgrid Using Consensus Protocol. IEEE Trans. Ind. Electron. 2020, 67, 1968-1979. [CrossRef]

18. Morstyn, T.; Savkin, A.V.; Hredzak, B.; Agelidis, V.G. Multi-Agent Sliding Mode Control for State of Charge Balancing Between Battery Energy Storage Systems Distributed in a DC Microgrid. IEEE Trans. Smart Grid 2018, 9, 4735-4743. [CrossRef] 
19. Olfati-Saber, R.; Murray, R.M. Consensus problems in networks of agents with switching topology and time-delays. IEEE Trans. Autom. Control 2004, 49, 1520-1533. [CrossRef]

20. Xiao, L.; Boyd, S.; Kim, S.-J. Distributed average consensus with least-mean-square deviation. J. Parallel Distrib. Comput. 2007, 67, 33-46. [CrossRef] 